\title{
HEART
}

\section{Left ventricular myocardial deformation and mechanical dyssynchrony in children with normal ventricular shortening fraction after anthracycline therapy}

Yiu-fai Cheung, Wen-jing Hong, Godfrey C F Chan, et al.

Heart 2010 96: 1137-1141 originally published online May 29, 2010

doi: $10.1136 /$ hrt.2010.194118

Updated information and services can be found at:

http:/heart.bmj.com/content/96/14/1137.full.html

\section{These include:}

References This article cites 31 articles, 13 of which can be accessed free at: http://heart.bmj.com/content/96/14/1137.full.html\#ref-list-1

Email alerting Receive free email alerts when new articles cite this article. Sign up in the service box at the top right corner of the online article.

Notes

To request permissions go to:

http://group.bmj.com/group/rights-licensing/permissions

To order reprints go to:

http://journals.bmj.com/cgi/reprintform

To subscribe to BMJ go to:

http://journals.bmj.com/cgi/ep 


\title{
Left ventricular myocardial deformation and mechanical dyssynchrony in children with normal ventricular shortening fraction after anthracycline therapy
}

\author{
Yiu-fai Cheung, Wen-jing Hong, Godfrey C F Chan, Sophia J Wong, Shau-yin Ha
}

Division of Paediatric Cardiology, Department of Paediatrics and Adolescent Medicine, Queen Mary Hospital, The University of Hong Kong, Hong Kong, China

\section{Correspondence to} Professor Yiu-fai Cheung, Division of Paediatric Cardiology, Department of Paediatrics and Adolescent Medicine, The University of Hong Kong, Queen Mary Hospital, 102 Pokfulam Road, Hong Kong, China; xfcheung@hkucc.hku.hk

Accepted 18 March 2010

\section{ABSTRACT}

Objective The M-mode-derived left ventricular shortening fraction is incorporated into most of the paediatric oncology protocols for monitoring of cardiotoxicity. This study tested the hypothesis that alteration of left ventricular myocardial deformation and mechanical dyssynchrony may occur in asymptomatic children after anthracycline therapy despite having left ventricular shortening fractions within the limits of normal.

Design Cross-sectional study.

Setting Tertiary paediatric cardiac centre.

Methods Left ventricular longitudinal, circumferential and radial myocardial deformation was determined using speckle tracking echocardiography in 45 patients aged $15.3 \pm 5.8$ years. Real-time three-dimensional echocardiographic data were acquired for the measurement of left ventricular volumes and systolic dyssynchrony index (SDI), the latter derived from the dispersion of time-to-minimum regional volume using a 16 -segment model. The results were compared with those of 44 controls

Results Compared with controls, patients had reduced left ventricular global systolic longitudinal strain $(p=0.012)$, circumferential strain $(p<0.001)$, radial strain $(p=0.006)$ and circumferential strain rate (SR; $p=0.002)$. The cumulative anthracycline dose correlated negatively with global longitudinal $(r=-0.33, p=0.027)$ and circumferential $(r=-0.32, p=0.035)$ SR. The left ventricular SDI was significantly greater in patients than controls $(4.46 \pm 1.52 \%$ vs $3.80 \pm 0.58 \%, p=0.03)$. The prevalence of left ventricular mechanical dyssynchrony (SDI $>4.96 \%$ ) in patients was $16 \%$ (95\% Cl $6 \%$ to $29 \%$ ). In patients, SDI correlated negatively with left ventricular ejection fraction $(r=-0.52, p<0.001)$, radial strain $(r=-0.35, p=0.021)$, circumferential strain $(r=-0.37$, $p=0.015)$ and circumferential SR $(r=-0.43, p=0.004)$, but not with the cumulative anthracycline dose ( $p=0.82$ )

Conclusions Impaired left ventricular myocardial deformation and mechanical dyssynchrony may exist in children after anthracycline therapy despite having normal left ventricular shortening fractions.

Whereas anthracyclines have been widely used for the treatment of childhood malignancies, the side-effect of cardiotoxicity remains an issue of concern. ${ }^{1-5}$ Left ventricular abnormalities are characterised by reduced ventricular mass, increased afterload and impaired contractility. ${ }^{3}$ Importantly, a recent study showed that even children who have received a cumulative doxorubicin dose as low as $45 \mathrm{mg} / \mathrm{m}^{2}$ have reduced left ventricular mass, ${ }^{3}$ implying the absence of a safe dose that is free of cardiotoxicity.

The M-mode-derived parameter of the left ventricular shortening fraction is incorporated into most of the paediatric oncology protocols for the monitoring of cardiotoxicity. ${ }^{4}$ This parameter is nonetheless limited to an assessment of radial contraction of two left ventricular segments, namely the mid-anteroseptal and mid-inferolateral segments. The importance of assessing not only the radial but also the longitudinal and circumferential myocardial deformation of the left ventricle has recently been increasingly acknowledged. ${ }^{6}$ Furthermore, segmental wall motion abnormalities found during and after anthracycline therapy may not be detectable by simply assessing the left ventricular shortening fraction. ${ }^{7}$ A shortening fraction that is within 'normal' limits may not thus always be reassuring.

The recently introduced speckle tracking echocardiography (STE) allows angle-independent quantification of myocardial deformation in different dimensions, ${ }^{8}$ while three-dimensional echocardiography has revolutionised the understanding of the synchronous contraction of different left ventricular segments in health and disease. ${ }^{9}$ In the present study, we tested the hypothesis that an alteration of left ventricular myocardial deformation and mechanical dyssynchrony may occur in asymptomatic children after anthracycline therapy despite having left ventricular shortening fractions within the limits of normal.

\section{METHODS \\ Subjects}

Forty-five children with acute lymphoblastic leukaemia who had been off treatment for at least 1 year and who had a left ventricular shortening fraction within normal limits ( $>28 \%$ ) were recruited. The cumulative dose of anthracyclines received and the surface ECG findings were noted The echocardiographic findings of these patients were compared with those of 44 control subjects, who were healthy siblings of patients and children with functional heart murmur, non-specific chest pain or palpitation attending the cardiac outpatient clinic, for which no underlying organic causes were identified. The body weight and height of all subjects were measured and the body surface area was calculated accordingly. 


\section{Conventional echocardiographic assessment}

All echocardiographic assessments were performed using the Vivid 7 ultrasound machine (GE Medical Systems, Horten, Norway). The average value of the echocardiographic indices from three cardiac cycles was used for analyses.

Standard M-mode assessment of the mid-left ventricular parasternal short axis was performed to derive the following parameters: left ventricular end-systolic and end-diastolic dimensions; thickness of interventricular septum and posterior left ventricular wall; left ventricular mass indexed for body surface area and shortening fraction. Pulsed-wave Doppler examination was performed to obtain peak mitral inflow velocities at early (E) and late (A) diastole and early deceleration time.

Tissue Doppler echocardiography was performed with the sample volume positioned at the basal left ventricular free wall-mitral annular junction to obtain the following: peak early (e) and late (a) diastolic myocardial tissue velocities, e/a ratio, and peak systolic myocardial tissue velocity (s).

\section{Speckle tracking echocardiography}

STE for the determination of left ventricular strain and strain rate (SR) was performed as reported previously. ${ }^{10}$ The fourchamber apical plane was used to assess left ventricular longitudinal strain and SR, whereas the short-axis plane at the level of the papillary muscles was used to assess left ventricular radial strain and circumferential strain and SR. Customised EchoPAC software (GE Medical Systems) was used to track speckles within the region of interest throughout the cardiac cycle for the derivation of systolic strain and SR. For quantification of regional myocardial deformation, the left ventricular free wall and ventricular septum were divided into three segments, whereas the left ventricular short-axis plane was divided into six segments. For the calculation of global longitudinal, circumferential and radial deformation, the entire traced left ventricular contours in the four-chamber and short-axis planes were used. We and others have previously reported on low intra and interobserver variability for the measurement of global longitudinal strain and SR. ${ }^{11} 12$

\section{Three-dimensional echocardiography}

Real-time three-dimensional echocardiographic imaging was performed from the apical view using a matrix array transducer (Vivid 7 ultrasound system; General Electric, Vingmed, Horten, Norway). Full-volume acquisition of the left ventricle was performed during breathhold. Offline analyses of three-dimensional datasets were performed using commercial four-dimensional analysis software (Tomtec Imaging Systems, Unterschleisheim, Germany). Quantitative analysis of the left ventricle involved the definition of two, three and four-chamber planes through the three-dimensional dataset at end-systole and end-diastole. The endocardial border was traced with a semi-automated detection process and a left ventricular cast was created for the derivation of left ventricular end-diastolic and end-systolic volumes and ejection fraction. By dividing this cast into pyramidal subvolumes based around a non-fixed central point, the time-volume data corresponding to each of the 16 standard myocardial segments, as defined by the American Society of Echocardiography, ${ }^{13}$ were obtained. A systolic dyssynchrony index (SDI), defined as the SD of the time taken to reach minimum regional volume for each of the segments as a percentage of the cardiac cycle, was calculated to quantify global left ventricular mechanical dyssynchrony. ${ }^{9}$ We have similarly reported low intra and interobserver variability for measurement of left ventricular SDI. ${ }^{14}$

\section{Statistical analysis}

All data are presented as means \pm SD. The demographic and echocardiographic parameters of patients were compared with those of control subjects using Student's t test. The left ventricular volumes were normalised by body surface area, and absolute values of systolic strain and SR were presented. Global left ventricular mechanical dyssynchrony was defined by left ventricular SDI exceeding the mean $+2 \mathrm{SD}$ as derived from control data. Pearson correlation analysis was used to assess relationships between deformation parameters and the cumulative anthracycline dose. A p value less than 0.05 is considered statistically significant. All statistical analyses were performed using SPSS version 11.5.

\section{RESULTS}

The 45 patients ( 28 males), aged $15.3 \pm 5.8$ years had all received anthracycline, doxorubicin or daunorubicin, as part of a chemotherapeutic regimen for the treatment of acute lymphoblastic leukaemia. The median cumulative anthracycline dose was $240 \mathrm{mg} / \mathrm{m}^{2}$ (range $120-470 \mathrm{mg} / \mathrm{m}^{2}$ ). None of the patients required cardiac irradiation. The patients were studied at a median of 6.3 years (range 2.7-19.8 years) after the last dose of anthracyclines. All of the patients had a sinus rhythm and none had prolongation of ORS duration. All patients were asymptomatic without the need for cardiac medications. The 44 controls (21 males) were aged $16.4 \pm 6.9$ years $(p=0.40)$. The weight $(49.7 \pm 16.6 \mathrm{~kg}$ vs $48.6 \pm 16.8 \mathrm{~kg}, \mathrm{p}=0.76)$ and body mass index $\left(21.5 \pm 4.9 \mathrm{~kg} / \mathrm{m}^{2}\right.$ vs $\left.19.9 \pm 4.0 \mathrm{~kg} / \mathrm{m}^{2}, \mathrm{p}=0.10\right)$ were similar between patients and controls. None of our patients had Doppler indices suggestive of left ventricular restrictive or relaxation abnormalities.

\section{Conventional echocardiographic parameters}

Table 1 summarises the echocardiographic findings in patients and controls. Albeit within the limits of normal, the left ventricular shortening fraction was slightly but significantly lower in patients than controls $(p=0.001)$. Compared with controls, patients had significantly greater left ventricular end-systolic

Table 1 Comparison of echocardiographic indices between patients and controls

\begin{tabular}{lccl}
\hline & $\begin{array}{l}\text { Patients } \\
(\mathbf{n}=\mathbf{4 5})\end{array}$ & $\begin{array}{l}\text { Controls } \\
(\mathbf{n}=\mathbf{4 4})\end{array}$ & p Value \\
\hline M-mode measurements & & & \\
LVDD (cm) & $4.6 \pm 0.5$ & $4.5 \pm 0.5$ & 0.30 \\
LVDS (cm) & $3.0 \pm 0.4$ & $2.8 \pm 0.4$ & $0.03^{*}$ \\
Shortening fraction (\%) & $34.5 \pm 3.3$ & $36.9 \pm 3.7$ & $0.001^{*}$ \\
Indexed left ventricular mass $\left(\mathrm{g} / \mathrm{m}^{2}\right)$ & $45.9 \pm 10.2$ & $54.1 \pm 11.9$ & $0.001^{*}$ \\
Mitral inflow Doppler indexes & & & \\
E (cm/s) & $100.0 \pm 19.9$ & $103.5 \pm 21.5$ & 0.49 \\
A (cm/s) & $52.2 \pm 12.6$ & $52.7 \pm 15.0$ & 0.87 \\
E/A ratio & $2.0 \pm 0.5$ & $2.1 \pm 0.6$ & 0.55 \\
E deceleration time (ms) & $130.3 \pm 18.2$ & $126.5 \pm 27.6$ & 0.45 \\
Mitral annular myocardial tissue velocities & & & \\
s (cm/s) & $9.8 \pm 2.0$ & $10.7 \pm 2.0$ & $0.037^{*}$ \\
e (cm/s) & $17.7 \pm 2.9$ & $18.0 \pm 2.3$ & 0.60 \\
a (cm/s) & $7.4 \pm 1.7$ & $7.7 \pm 2.4$ & 0.51 \\
e/a ratio & $2.5 \pm 0.7$ & $2.5 \pm 0.6$ & 0.95 \\
\hline A $\mathrm{seak}$.
\end{tabular}

A, peak mitral inflow velocity at late diastole; a, mitral annular late diastolic myocardial tissue velocity; $E$, peak mitral inflow velocity at early diastole; e, mitral annular early diastolic myocardial tissue velocity; LVDD, left ventricular dimension at diastole; LVDS, left ventricular dimension at systole; $\mathbf{s}$, mitral annular systolic myocardial tissue velocity. *Statistically significant. 
dimension $(p=0.001)$, decreased indexed left ventricular mass $(p=0.001)$ and reduced mitral annular systolic myocardial velocity $(p=0.037)$. On the other hand, the mitral diastolic inflow velocities and mitral annular diastolic myocardial tissue velocity were similar between patients and controls (all p>0.05).

\section{Myocardial strain and SR}

The left ventricular longitudinal, radial and circumferential regional systolic strain parameters of patients and controls are shown in figure 1. The longitudinal systolic strain of the left ventricular free wall was similar between patients and controls, whereas that of the basal $(p=0.03)$ and mid $(p=0.03)$ septum was lower in patients. The systolic radial strain of all of the six segments was significantly lower in patients than controls (all $p<0.05)$. The segmental circumferential systolic strain was lower for the anteroseptal $(p<0.001)$, inferoseptal $(p=0.008)$, inferior $(p=0.001)$ and anterior $(p=0.001)$ segments in patients than controls.

Taking into account the deformation of all the regional segments, the global systolic longitudinal $(17.6 \pm 3.0 \%$ vs $19.0 \pm 2.2, p=0.012)$, circumferential $(14.5 \pm 2.9 \%$ vs $17.4 \pm 4.3$, $\mathrm{p}<0.001)$ and radial $(40.1 \pm 15.6 \%$ vs $50.0 \pm 16.4 \%, p=0.006)$ strain was significantly lower in patients than controls.

With regard to $S R$, compared with controls, patients had similar longitudinal $(0.95 \pm 0.18 / \mathrm{s}$ vs $1.00 \pm 0.15 / \mathrm{s}, \mathrm{p}=0.16)$ but lower circumferential systolic SR $(0.90 \pm 0.21 / \mathrm{s}$ vs $1.06 \pm 0.28 / \mathrm{s}$, $\mathrm{p}=0.002$ ).

Of the global strain and SR parameters in patients, the cumulative anthracycline dose correlated significantly with global longitudinal $(r=-0.33, p=0.027)$ and circumferential $(r=-0.32, p=0.035) S R$, whereas the time from completion of chemotherapy correlated with global longitudinal strain $(\mathrm{r}=-0.33, \mathrm{p}=0.032)$ and SR $(\mathrm{r}=-0.33, \mathrm{p}=0.03)$.

\section{Three-dimensional global left ventricular mechanical dyssynchrony}

The left ventricular SDI was significantly greater in patients than controls $(4.46 \pm 1.52 \%$ vs $3.80 \pm 0.58 \%, p=0.03)$. Based on control data, global left ventricular mechanical dyssynchrony was defined as SDI greater than $4.96 \%$. The prevalence of left ventricular systolic dyssynchrony in patients was $16 \%$ (seven of 45) (95\% CI $6 \%$ to $29 \%$ ). Figure 2 shows examples of synchronous and dyssynchronous contraction of different left ventricular segments during systole in a control and a patient, respectively.

In patients, SDI correlated negatively with three-dimensional echocardiography-derived left ventricular ejection fraction $(\mathrm{r}=-0.52, \mathrm{p}<0.001)$, global systolic radial $(\mathrm{r}=-0.35, \mathrm{p}=0.021)$ and circumferential $(\mathrm{r}=-0.37, \mathrm{p}=0.015)$ strain and global circumferential systolic SR ( $r=-0.43, p=0.004)$, but not with age $(p=0.1)$, left ventricular shortening fraction $(p=0.58)$, global longitudinal strain $(p=0.24)$ and SR $(p=0.22)$, time from completion of chemotherapy ( $p=0.82$ ) or the cumulative dose of anthracyclines $(p=0.82)$. Multiple regression analysis was used to identify significant correlates of SDI in patients. The dependent variables entered were those found to be significant by univariate analysis as listed above. The left ventricular ejection fraction was identified to be the only significant correlate $(\beta=-0.51, p<0.001)$.

\section{DISCUSSION}

Our findings suggest that children after anthracycline therapy, albeit having left ventricular shortening fraction within normal
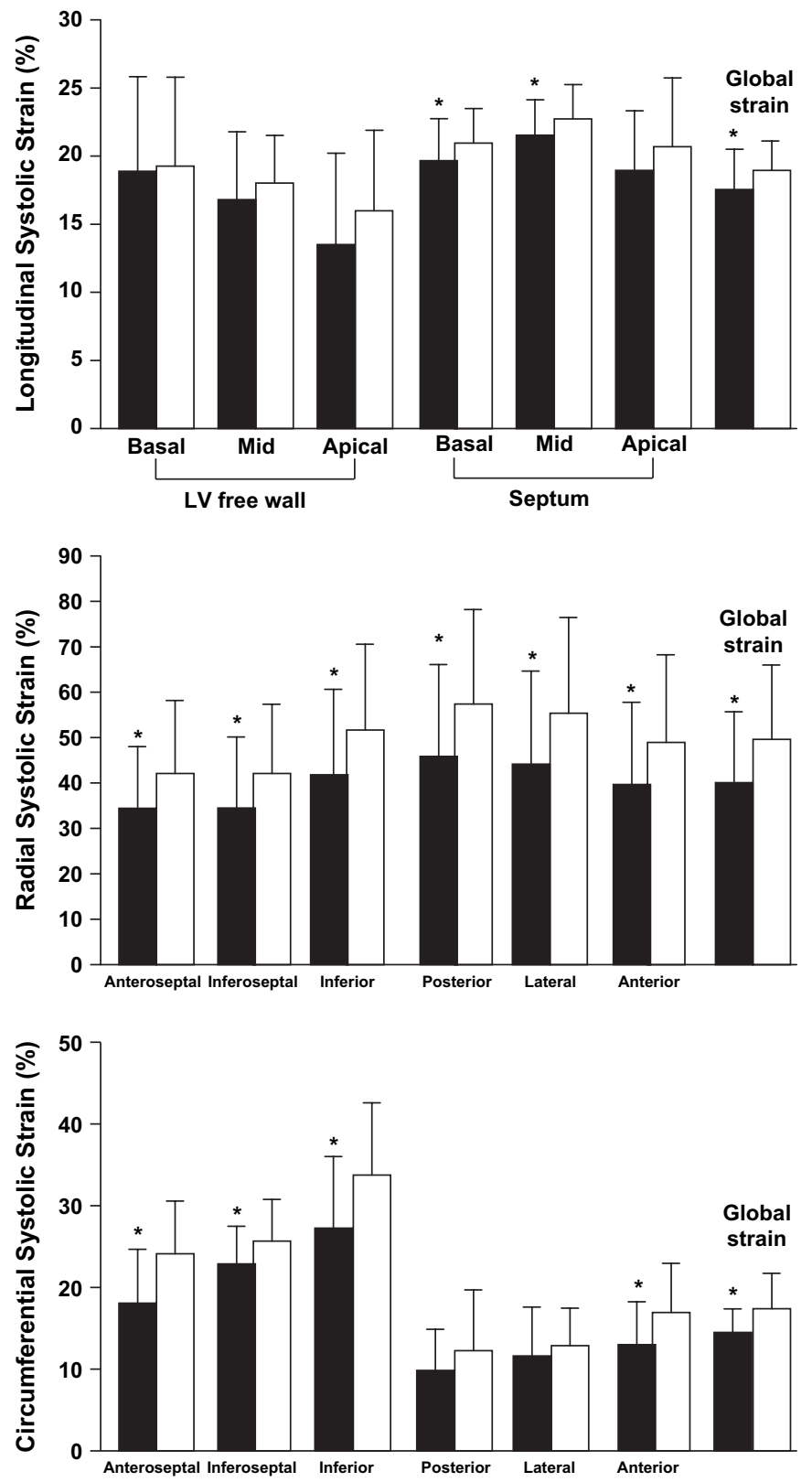

Figure 1 Comparisons of regional systolic longitudinal (upper panel), radial (middle panel), and circumferential (lower panel) strain between patients (black columns) and controls (white columns) $\left({ }^{*} p<0.05\right.$ vs controls). LV, left ventricular.

limits, may have reduced left ventricular myocardial deformation in all of the three dimensions. Importantly, dyssynchronous left ventricular contraction was documented for the first time using three-dimensional echocardiography in these children, with a prevalence of $16 \%$ in our cohort. The present study represents a paradigm shift in the monitoring of anthracycline cardiotoxicity from conventional assessment of changes in the left ventricular cavity dimension to direct assessment of magnitude, rate and synchronicity of myocardial deformation.

While frequently incorporated into paediatric oncology protocols for the monitoring of left ventricular systolic function, ${ }^{4}$ the $\mathrm{M}$-mode-derived shortening fraction has its limitations. Derivation of the left ventricular shortening fraction depends on the method used to obtain left ventricular dimensions, ${ }^{15}$ and values can vary considerably between different observers. ${ }^{16}$ Additional issues include assessment being limited 

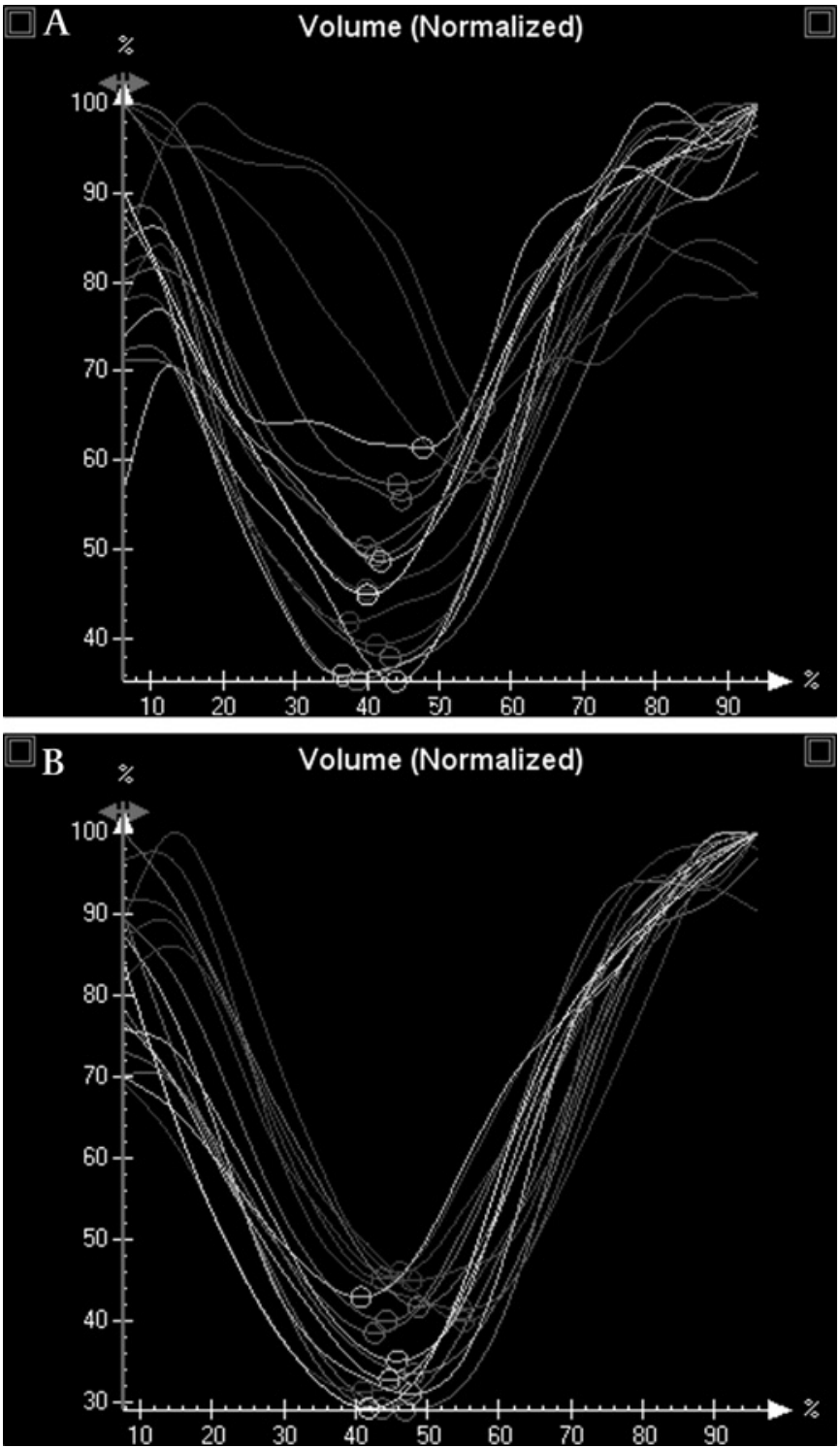

Figure 2 Regional volume curves, normalised to individual maximum, demonstrating $(A)$ dyssynchronous contraction of multiple segments in a patient and (B) synchronous contraction in the 16 left ventricular segments in a control.

to the radial contraction of two myocardial segments and load dependency. The incorporation of end-systolic wall stress in the evaluation provides a means to adjust for the afterload, although this has not been widely adopted in clinical practice. ${ }^{4}$ In patients who may otherwise be regarded as having normal left ventricular systolic function based on isolated M-mode findings, the new echocardiographic techniques have unmasked evidence of left ventricular systolic dysfunction, as characterised by reduced and dyssynchronous systolic myocardial deformation.

Increasing data suggest that systolic strain and SR are strong indices of ventricular contractility. ${ }^{17-19}$ It is worth noting that the peak systolic SR, which represents the rate of myocardial deformation, may even better reflect the rate of myocardial force generation and hence contractility. ${ }^{18} 20$ Ganame et al ${ }^{21}$ have reported on the reduction of predominantly longitudinal regional strain and SR as determined by tissue Doppler imaging in children after low-dose anthracycline $\left(\leq 300 \mathrm{mg} / \mathrm{m}^{2}\right)$ therapy. Nonetheless, the limitations of tissue Doppler imaging include angle dependency, limitation of assessment of myocardial deformation to one dimension and susceptibility to noisy artefacts. $^{22}{ }^{23}$ By contrast, STE has the advantages of angle independence, ${ }^{24}$ the generation of more reproducible SR profiles, ${ }^{11}$ and allowing comprehensive assessment of both regional and global left ventricular myocardial deformation in three dimensions. The importance of assessing not only longitudinal but also radial and circumferential myocardial deformation of the left ventricle is increasingly acknowledged. ${ }^{6}$ Our findings suggest that indices of radial and circumferential, in contrast to longitudinal, deformation are perhaps more sensitive in the detection of left ventricular systolic dysfunction after anthracycline therapy (figure 1). Indeed, the more sensitive nature of circumferential deformation has been shown in adults with myocardial ischaemia. $^{25}$

Notwithstanding the lack of data on left ventricular mechanical dyssynchrony in patients after anthracycline therapy, two recent case reports suggested a favourable effect of cardiac resynchronisation therapy (CRT) on anthracyline-induced cardiotoxicity in patients with normal ORS duration. The first described the prompt improvement of left ventricular ejection fraction in a 9-year-old girl who developed acute heart failure during chemotherapy for acute myeloid leukaemia, ${ }^{26}$ whereas the second reported on the normalisation of left ventricular ejection fraction in a 46-year-old woman who developed severe heart failure after completion of chemotherapy for breast cancer. ${ }^{27}$ In another small series of four adults with doxorubicin-induced cardiomyopathy in association with left bundle branch block and prolonged ORS duration, the impact of CRT-induced left ventricular reverse remodelling was reported to sustain over a mean follow-up duration of 18.5 months. ${ }^{28}$ Although these anecdotal reports appear encouraging, the prevalence, correlates and clinical implication of left ventricular mechanical dyssynchrony in patients after anthracyline therapy remain unclear. To our knowledge, this is the first study to explore mechanical dyssynchrony by three-dimensional echocardiography in children after anthracycline therapy.

In our asymptomatic patient cohort, the finding of a $16 \%$ prevalence of left ventricular mechanical dyssynychrony is intriguing. The abnormal SDI has clinical implications as shown by its negative correlations with left ventricular ejection fraction and myocardial deformation in the circumferential and radial dimensions. Similar to the two reported patients, ${ }^{26}{ }^{27}$ our patients had normal ORS duration and absence of left bundle branch block. Therefore, electrical dyssynchrony is unlikely to be the culprit. Multifocal areas of patchy interstitial fibrosis are characteristic features of myocardial biopsies from patients with anthracycline cardiomyopathy. ${ }^{29}$ An ovine model of anthracycline-induced cardiotoxicity has revealed the heterogeneous distribution of left ventricular fibrosis based on histology and late gadolinium enhancement on cardiac magnetic resonance. ${ }^{30}$ Segmental fibrosis of the left ventricle in vivo has indeed been suggested by subendocardial late gadolinium enhancement in adults with anthracycline cardiotoxicity following treatment for Ewing's sarcoma. ${ }^{31}$ It is possible, albeit speculative, that non-uniform alteration of left ventricular myocardium by anthracycline may contribute to left ventricular mechanical dyssynchrony. The magnitude of mechanical dyssynchrony is not, however, related to the cumulative anthracycline dose or the time from completion of chemotherapy. Taken together, the present findings can perhaps provide further rationale for the consideration of CRT in patients with refractory left ventricular failure after anthracycline therapy.

In the present study, we have limited cardiac assessment to patients who had been off treatment for at least 1 year. Further application of these new techniques in echocardiographic 
surveillance during the administration of chemotherapy might facilitate the early detection of myocardial dysfunction for timely intervention. Longitudinal studies are nonetheless required to provide evidence as to whether this approach would alter the course of anthracycline-induced cardiomyopathy.

Several limitations to this study warrant comment. The crosssectional nature of the present study does not provide information on the value of myocardial deformation and dyssynchrony parameters in prognostication, an issue that requires clarification by longitudinal studies. Second, we have not calculated the global radial SR, as averaging of the radial SR of the six segments is not feasible with the present version of the software. Third, although STE allows interrogation of global strain and SR parameters in the three dimensions, these parameters are not entirely load dependent and need to be interpreted with caution when an alteration of cardiac status with acute changes in load is anticipated. Changes in load are of particular relevance for our group of patients, given the well-documented finding of increased afterload secondary to decreased wall thickness and left ventricular mass, ${ }^{13} 32$ which have been demonstrated to progress with time. ${ }^{3}$

In conclusion, a normal left ventricular shortening fraction in children after anthracycline therapy may not be completely reassuring, and an assessment of the magnitude, rate and synchronicity of myocardial deformation may provide additional insights on the alteration of left ventricular mechanics.

Funding This study is supported by the Children's Cancer Foundation and CRCG funding, the University of Hong Kong.

Competing interests None

Ethics approval This study was conducted with the approval of the institutional review board of the University of Hong Kong/Hospital Authority Hong Kong West Cluster, Hong Kong.

Provenance and peer review Not commissioned; externally peer reviewed.

\section{REFERENCES}

1. Lipshultz SE, Colan SD, Gelber RD, et al. Late cardiac effects of doxorubicin therapy for acute lymphoblastic leukemia in childhood. N Engl J Med 1991;324:808-15.

2. Lipshultz SE, Lipsitz SR, Mone SM, et al. Female sex and drug dose as risk factors for late cardiotoxic effects of doxorubicin therapy for childhood cancer. N Engl J Med 1995;332:1738-43.

3. Lipshultz SE, Lipsitz SR, Sallan SE, et al. Chronic progressive cardiac dysfunction years after doxorubicin therapy for childhood acute lymphoblastic leukemia. J Clin Oncol 2005;23:2629-36.

4. van Dalen EC, van den Brug M, Caron HN, et al. Anthracycline-induced cardiotoxicity: comparison of recommendations for monitoring cardiac function during therapy in paediatric oncology trials. Eur J Cancer 2006;42:3199-205.

5. Elbl L, Hrstkova H, Tomaskova I, et al. Long-term serial echocardiographic examination of late anthracycline cardiotoxicity and its prevention by dexrazoxane in paediatric patients. Eur J Pediatr 2005;164:678-84.

6. Arita T, Sorescu GP, Schuler BT, et al. Speckle-tracking strain echocardiography for detecting cardiac dyssynchrony in a canine model of dyssynchrony and heart failure. Am J Physiol Heart Circ Physiol 2007:293:H735-42.

7. Kapusta L, Groot-Loonen J, Thijssen JM, et al. Regional cardiac wall motion abnormalities during and shortly after anthracycline therapy. Med Pediatr Oncol 2003:41:426-35.

8. Leitman M, Lysyansky $P$, Sidenko $S$, et al. Two-dimensional strain-a novel software for real-time quantitative echocardiographic assessment of myocardial function. J Am Soc Echocardiogr 2004;17:1021-9.

9. Kapetanakis $\mathbf{S}$, Kearney MT, Siva A, et al. Real-time three-dimensional echocardiography: a novel technique to quantify global left ventricular mechanical dyssynchrony. Circulation 2005;112:992-1000.
10. Cheung EW, Liang XC, Lam WW, et al. Impact of right ventricular dilation on left ventricular myocardial deformation in patients after surgical repair of tetralogy of Fallot. Am J Cardiol 2009;104:1264-70.

11. Chow PC, Liang XC, Cheung EW, et al. New two-dimensional global longitudinal strain and strain rate imaging for assessment of systemic right ventricular function. Heart 2008:94:855-9.

12. Mavinkurve-Groothuis AM, Weijers G, Groot-Loonen, et al. Interobserver, intraobserver and intrapatient reliability scores of myocardial strain imaging with 2-D echocardiography in patients treated with anthracyclines. Ultrasound Med Biol 2009;35:697-704.

13. Cerqueira MD, Weissman NJ, Dilsizian V, et al. Standardized myocardial segmentation and nomenclature for tomographic imaging of the heart: a statement for healthcare professionals from the Cardiac Imaging Committee of the Council on Clinical Cardiology of the American Heart Association. Circulation 2002;105:539-42.

14. Liang XC, Cheung EW, Wong SJ, et al. Impact of right ventricular volume overload on three-dimensional global left ventricular mechanical dyssynchrony after surgical repair of tetralogy of Fallot. Am J Cardiol 2008;102:1731-6.

15. Sahn DJ, DeMaria A, Kisslo J, et al. Recommendations regarding quantitation in M-mode echocardiography: results of a survey of echocardiographic measurements. Circulation 1978;58:1072-83.

16. Lipshultz SE, Easley KA, Orav EJ, et al. Reliability of multicenter pediatric echocardiographic measurements of left ventricular structure and function: the prospective P(2)C(2) HIV study. Circulation 2001;104:310-16.

17. Greenberg NL, Firstenberg MS, Castro PL, et al. Doppler-derived myocardial systolic strain rate is a strong index of left ventricular contractility. Circulation 2002;105:99-105

18. Pellerin D, Sharma R, Elliott $P$, et al. Tissue Doppler, strain, and strain rate echocardiography for the assessment of left and right systolic ventricular function. Heart 2003;89(Suppl 3):iii9-17

19. Weidemann $\mathbf{F}$, Jamal F, Kowalski $M$, et al. Can strain rate and strain quantify changes in regional systolic function during dobutamine infusion, B-blockade, and atrial pacing -implications for quantitative stress echocardiography. J Am Soc Echocardiogr 2002;15:416-24

20. Weidemann F, Jamal F, Sutherland GR, et al. Myocardial function defined by strain rate and strain during alterations in inotropic states and heart rate. Am J Physiol Heart Circ Physiol 2002;283:H792-9.

21. Ganame J, Claus P, Uyttebroeck A, et al. Myocardial dysfunction late after low-dose anthracycline treatment in asymptomatic pediatric patients. J Am Soc Echocardiog 2007:20:1351-8.

22. Marwick TH. Measurement of strain and strain rate by echocardiography: ready for prime time? J Am Coll Cardiol 2006;47:1313-27.

23. $\mathbf{~ N g ~ A C , ~ T r a n ~ d a ~ T , ~ N e w m a n ~ M , ~ e t ~ a l . ~ C o m p a r i s o n ~ o f ~ m y o c a r d i a l ~ t i s s u e ~ v e l o c i t i e s ~}$ measured by two-dimensional speckle tracking and tissue Doppler imaging. Am J Cardiol 2008; 102:784-9.

24. Amundsen BH, Helle-Valle T, Edvardsen T, et al. Noninvasive myocardial strain measurement by speckle tracking echocardiography: validation against sonomicrometry and tagged magnetic resonance imaging. J Am Coll Cardiol 2006;47:789-93.

25. Tanaka H, Oishi Y, Mizuguchi $Y$, et al. Three-dimensional evaluation of dobutamineinduced changes in regional myocardial deformation in ischemic myocardium using ultrasonic strain measurements: the role of circumferential myocardial shortening. J Am Soc Echocardiogr 2007;20:1294-9.

26. Jones BO, Davis AM, Alison J, et al. Cardiac re-synchronization therapy in a child with severe anthracycline-induced congestive heart failure and normal QRS duration. $\checkmark$ Heart Lung Transplant 2007;26:1333-5.

27. Ahlehoff 0, Galloe AM, Hansen PR. Anthracycline-induced cardiomyopathy: favourable effects of cardiac resynchronization therapy. Int J Cardiol. Published Online First: 8 Jan 2009. doi: 10.1016/j.ijcard.2008.11.194.

28. Ajijola $\mathbf{O A}$, Nandigam KV, Chabner BA, et al. Usefulness of cardiac resynchronization therapy in the management of doxorubicin-induced cardiomyopathy. Am J Cardiol 2008;101:1371-2.

29. Takemura G, Fujiwara H. Doxorubicin-induced cardiomyopathy from the cardiotoxic mechanisms to management. Prog Cardiovasc Dis 2007;49:330-52.

30. Psaltis PJ, Carbone A, Nelson AJ, et al. An ovine model of anthracycline-induced cardiotoxicity - applicability as a pre-clinical surrogate for nonischaemic cardiomyopathy. Heart Lung Circ 2009;18S:S169.

31. Perel RD, Slaughter RE, Strugnell WE. Subendocardial late gadolinium enhancement in two patients with anthracycline cardiotoxicity following treatment for Ewing's sarcoma. J Cardiovasc Magn Reson 2006;8:789-91.

32. Sorensen K, Levitt G, Sebag-Monteflore D, et al. Cardiac function in Wilm's tumo survivors. J Clin Oncol 1995;13:1546-56. 